\title{
Bio-inspired ciliary force sensor for robotic platforms
}

\author{
Pedro Ribeiro ${ }^{1,2}$, Mohammed Asadullah Khan ${ }^{3}$, Ahmed Alfadhel ${ }^{3}$, Jürgen Kosel ${ }^{3}$, Fernando Franco ${ }^{1,2}$, \\ Susana Cardoso ${ }^{1,2}$, Alexandre Bernardino ${ }^{4}$, Alexander Schmitz ${ }^{5}$, José Santos-Victor ${ }^{4}$ and Lorenzo Jamone ${ }^{6,4}$
}

\begin{abstract}
The detection of small forces is of great interest in any robotic application that involves interaction with the environment (e.g. objects manipulation, physical human-robot interaction, minimally invasive surgery), since it allows the robot to detect the contacts early on and to act accordingly. In this work, we present a sensor design inspired by the ciliary structure frequently found in nature, consisting of an array of permanently magnetized cylinders (cilia) patterned over a giant magnetoresistance sensor (GMR). When these cylinders are deformed in shape due to applied forces, the stray magnetic field variation will change the GMR sensor resistivity, thus enabling the electrical measurement of the applied force. In this paper we present two $3 \times 3 \mathrm{~mm}^{2}$ prototypes composed of an array of 5 cilia with $1 \mathrm{~mm}$ of height and $120 \mu \mathrm{m}$ and $200 \mu \mathrm{m}$ of diameter for each prototype. A minimum force of $333 \mu \mathrm{N}$ was measured. A simulation model for determining the magnetized cylinders average stray magnetic field is also presented.
\end{abstract}

Index Terms-Biomimetics, Force and tactile sensing, Soft Materials Robotics

\section{INTRODUCTION}

$\mathbf{T}$ ACTILE sensing is a pivotal part of the development of intelligent robots, allowing such machines to feel the surrounding environment, and providing crucial support for the execution of most robotic tasks. However despite the increasing interest in the development of tactile sensing solutions during the last forty years, these tecnologies remain

Manuscript received: September, 10, 2016; Revised December, 4, 2016; Accepted January, 6, 2017.

This paper was recomended for publication by Editor John Wen upon evaluation of the Associate Editor and Reviewers' comments. This work was partially supported by EXCL/CTM-NAN/0441/2012, PTDC/CTMNAN/3146/2014 and UID/EEA/50009/2013 projects. F. Franco acknowledges FCT for the grant SFRH/BD/111538/2015. L. Jamone acknowledges project LIMOMAN - PIEFGA-2013-628315.

${ }^{1}$ INESC - Microsistemas e Nanotecnologias and IN, Lisbon, Portugal, pedro.q.ribeiro@tecnico.ulisboa.pt; scardosolinesc-mn.pt; ffrancolinesc-mn.pt

${ }^{2}$ Department of Physics, Instituto Superior Técnico, Lisbon, Portugal

${ }^{3}$ Computer, Electrical and Mathematical Sciences and Engineering Division (CEMSE), King Abdullah University of Science and Technology (KAUST), Thuwal, Saudi Arabia, ahmed.fadhel@kaust.edu.sa; jurgen.kosel@kaust.edu.sa

${ }^{4}$ Instituto de Sistemas e Robótica, Instituto Superior Técnico, Lisbon, Portugal, alex@ist.ist.utl.pt; jasveisr.ist.utl.pt

${ }^{5}$ Department of Modern Mechanical Engineering, School of Creative Science and Engineering, Waseda University, Tokyo, Japan, schmitzlaoni.waseda.jp

${ }^{6}$ ARQ (Advanced Robotics at Queen Mary), School of Electronic Engineering and Computer Science, Queen Mary University of London, United Kingdom, l. jamone dqmul.ac.uk

Digital Object Identifier (DOI): see top of this page

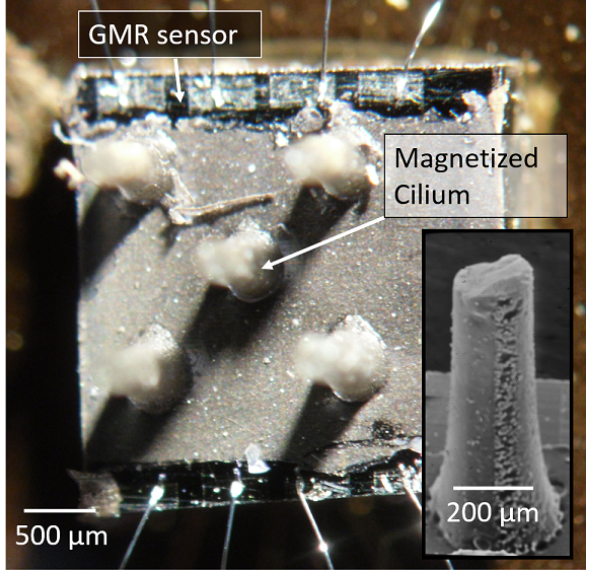

Fig. 1: Photograph of the finished sensor, with $200 \mu \mathrm{m}$ diameter and $1 \mathrm{~mm}$ height cilia (nominal). Note that the GMR sensor is comprised by the whole area under the cilia. Inset: Scanning electron microscope photograph of a cilium of the same sensor.

largely under-developed, especially for what concerns reliability, sensitivity and miniaturization [1] [2].

The advancements of micro-fabrication techniques fueled by the microelectronics industry, as well as the discovery of novel materials, have sparked, since the early 2000 s, a renewed interest in developing highly miniaturized and reliable tactile sensors, easier to integrate in distributed environments [3]. Existing sensors explore practically all methods of transduction, like piezoresistive [4] [5], capacitive [6], optical [7] [8], piezoelectric [9] and magnetic field based sensors [10] [11]. For instance, optical sensors are characterized by having good sensitivities and high spatial resolutions, but are power hungry and bulky [7].

However, most of these sensors operate within the $1 \mathrm{mN}$ to $10 \mathrm{~N}$ (or more) range, and therefore a novel sensing solution that could measure forces lower than $1 \mathrm{mN}$ could nicely complement the existing technologies, especially in applications in which extreme precision and movement control is a requirement, e.g. microsurgery. The development of robotic assistance for this type of surgery has been a recent research topic, with the implementation of tactile sensors being one of the main challenges, since sub-mN resolution sensors must be fitted in an instrument with an available area in the order of 1 $\mathrm{mm}^{2}$ for sensors, to ensure the surgical procedure safety [12].

One of the tactile sensing structures often found in nature 

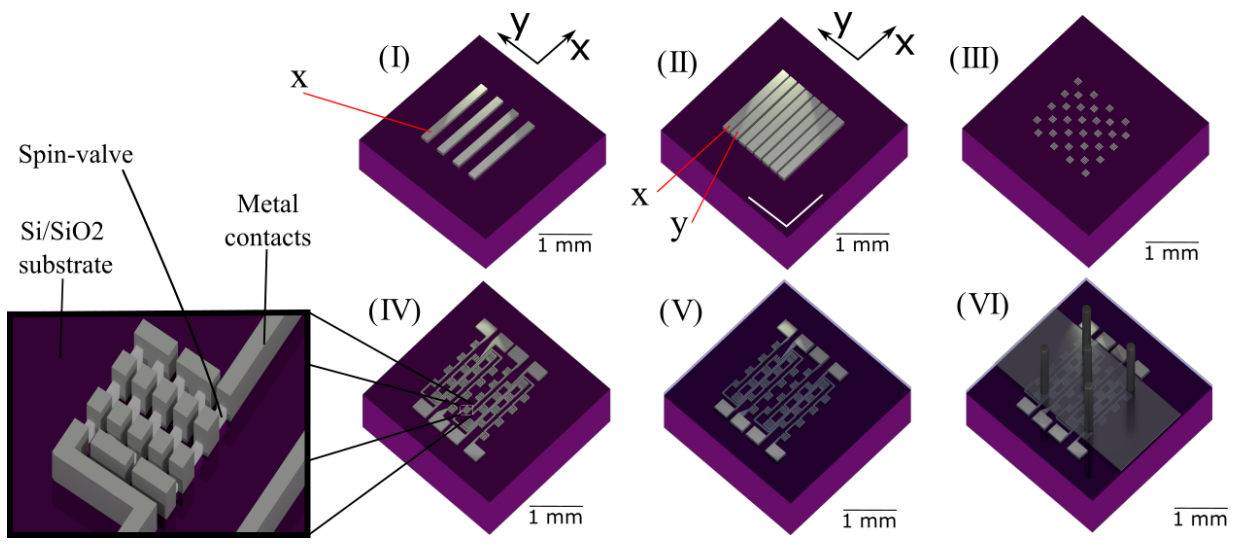

Fig. 2: Structures obtained after each fabrication step. I, II) deposition of both multilayered batches to obtain sensitivity in both substrate plane directions. The sensitive direction of each deposition is indicated with $x$ or $y$ III) Spin-valve patterning process IV) Deposition of the metallic contacts to create the spin-valve element arrays and definition of the contact pads. V) Deposition of the passivation layer VI) Patterning and deposition of the magnetized artificial cilia array to the sensor.

is the ciliary, commonly found in insects. This structure is composed of multiple mechano-receptors, each one constituted by a passive organelle called cilium (an elongated hair-like protuberance) coupled to an active organelle called dendrite (a nervous system ending that sends electrical pulses whenever the cilium is actuated) [13].

Some other solutions that take advantage of the ciliary structure emerged for air or water flow detection, using as transduction method the capacitive [14], or piezoelectric properties of a pillar composed by this type of material [15]. However, these solutions use rigid materials as cilia, which can be used to detect light induced forces, but can be easily deformed (or broken) by slightly larger forces, making them unsuitable for integration with other sensing tecnologies operating within a higher force magnitude range.

To overcome this limitation, elastomers can be used, as reported in [16], where an elastomeric compound with embedded magnetized nanoparticles was grown over a magnetic field sensor, creating an array of cilia structures. However this method only allows control of the rigidity of the fabricated cilia, and not of their number, position and dimensions.

By microfabricating the elastic cilia, this limitation can be overcome, as shown recently in a sensor used to detect flow speeds in microfluidics setups [17], and as a proof of concept in detecting force [18].

In those works focus was put on achieving high spatial resolutions (in the order of $0.01 \mathrm{~mm}^{2}$ ) with very small but distributed sensitive areas, while sacrificing measuring range and sensitivity. However, the total area of the sensor was in the order of $10 \mathrm{~cm}^{2}$. In the present work, we propose and report the fabrication of a miniaturized sensor, weighting 20 $\mathrm{mg}$, and spanning over an area of $3 \times 3 \mathrm{~mm}^{2}$ of which $4 \mathrm{~mm}^{2}$ is the sensitive area, to achieve a higher GMR sensor resolution (see Fig. 1). The manufactured sensor was able to discriminate a minimum force of $333 \mu \mathrm{N}$ roughly corresponding to the weight of a $2 \times 2 \mathrm{~cm}$ piece of normal paper, although lower forces should be detectable. Furthermore we present a simulation model able to predict the behaviour of cilia deformation under applied forces and the respective stray magnetic fields emitted by these structures.

\section{Methodology}

To measure the applied force, a giant magnetoresistance (GMR) sensor operating with a DC current input serves as the substrate for an artificial cilia array (see Fig. 1). When an external force is applied in a certain direction, the artificial cilia geometry deformation caused by this force will modify the stray field emanating from it, thus changing the resistivity of the underlying sensor (see Fig. 3). The sensitivity and measuring range of the sensor can be engineered by changing the characteristics of the deposited cilia length, diameter and Young's Modulus. The ciliary sensor fabrication is composed of two major steps:

1) Fabrication of the GMR sensor[19]

2) Fabrication of the artificial cilia and insertion over the GMR sensor[17]

The fabrication scheme is presented in Fig. 2.

\section{A. GMR sensor fabrication}

The GMR sensor used was fabricated at the clean room facilities of INESC-MN at Lisbon and consisted of five lithographic steps and five deposition steps, over a $\mathrm{Si}$ substrate with a $1500 \AA \mathrm{SiO}_{2}$ layer. The process used to fabricate these sensors is similar to the one used to fabricate hard-drive read heads, making the possibility of mass-producing this type of sensor in an industrial setup possible.

The first two steps consisted in performing standard lithography to define the substrate areas where the spin-valve stack (a multi-layered structure of the following thin metallic films, from bottom to top: Ta $10 \AA / \mathrm{NiFe} 28 \AA / \mathrm{CoFe} 25 \AA / \mathrm{Cu} 26$ $\AA / \mathrm{CoFe} 23 \AA / \mathrm{MnIr} 80 \AA /$ Ta $30 \AA$ ) would be placed, followed by its deposition. Since the produced sensor was meant to be sensitive in both directions of the substrate plane, a second deposition was necessary to deposit a spin-valve stack with a sensitive direction perpendicular to the previous one. Both these processes were performed using an Ion Beam Sputtering system [20]. 
In order to obtain a linear response from the magnetic sensor, the material deposited in the previous steps must be patterned into smaller $60 \times 3 \mu \mathrm{m}^{2}$ active sensing elements, by performing a Ion Beam Milling step [20] over the sample.

The metallic contacts to connect the spin-valve series, as well as to create the contact pads, were defined by lift-off after the deposition of a $3000 \AA \mathrm{Al}_{98.6} \mathrm{Si}_{1.0} \mathrm{Cu}_{0.4}$ thin film followed by a $150 \AA \mathrm{TiW}(\mathrm{N})$ thin film for oxidation protection, using DC magnetron [20] sputtering.

Finally, to achieve further protection from external elements, electrical insulation and good adhesion of the structures to be deposited in the next step, a double insulation layer composed of $\mathrm{Al}_{2} \mathrm{O}_{3} 2000 \AA / \mathrm{SiO}_{2} 2000 \AA$ was deposited in order to cover the whole sample, except in the regions where the contact pads are located.

The finished sensors were measured to determine their sensitivity and linear range, determined to be $53 \Omega \mathrm{Oe}^{-1}$ and -20 Oe to 20 Oe respectively.

\section{B. Artificial cilia fabrication}

The magnetized cilia were fabricated at KAUST in Saudi Arabia. The cilia nanocomposite is made by mixing $\mathrm{NdFeB}$ magnetic beads with a $5 \mu \mathrm{m}$ nominal diameter, with polydimethylsiloxane (PDMS) (Sylgard 184 Silicone Elastomer, Dow Corning Corporation) being used as the polymeric matrix. A $16 \%$ weight ratio concentration of magnetic beads to PDMS was used to provide a high magnetization in volume of the artificial cilia, without compromising the intrinsic elastic properties of the PDMS. To insert the cilia over the sensor, a $1 \mathrm{~mm}$ thick Poly(methyl methacrylate) (PMMA) layer was used as a master mold where $200 \mu \mathrm{m}$ and $120 \mu \mathrm{m}$ holes were patterned using a $\mathrm{CO}_{2}$ laser cutter.

The mold is then placed over the sample, after applying a thin $2 \mu \mathrm{m}$ PDMS layer over the sensor to promote adhesion onto the $\mathrm{SiO}_{2}$ film, and the composite is poured into mold, and cured at $90^{\circ} \mathrm{C}$ for 1 hour. After releasing the mold from the cured structure, the magnetic beads were magnetized in the direction of the cilium symmetry axis by applying a $10 \mathrm{kOe}$ magnetic field.

\section{Simulation}

As referred in section II, the response of the sensor is highly dependent on the artificial cilia characteristics (both intrinsic and extrinsic), and to a lesser extent, to the GMR sensor behaviour. In this sensor there are two major physics at stake about which would be important to have a reliable behavioural prediction (see Fig. 3):

- The structural mechanics of the artificial cilia (namely, how they deform under a certain type of load)

- The stray field generated for each deformation

\section{A. Artificial Cilia structural mechanics}

A numerical method was used to model the cilia, since the pillars dimensions (with diameter to length ratios lower than 1:10) cannot be modelled by classical beam theories [21]. To quantitatively investigate the effect of dimension

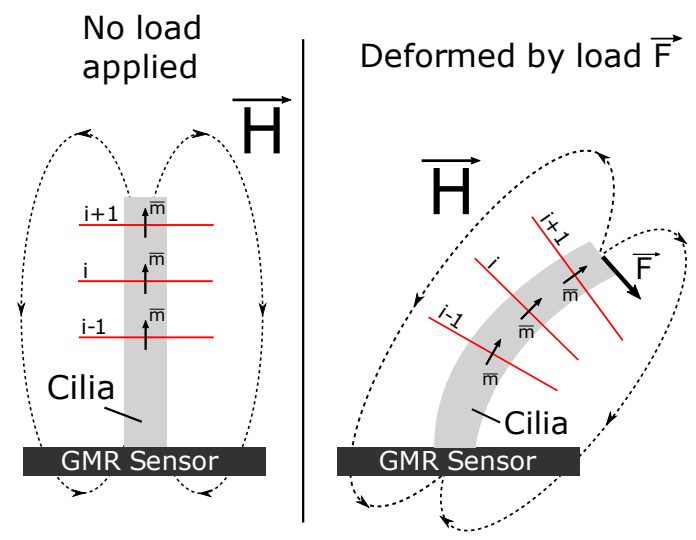

Fig. 3: Diagram of the pillar deformation model used to determine its magnetization. Left, the pillar is non-deformed and all dipoles point in the direction of the pillar long axis. Right, the pillar suffered a deformation and the dipoles rotated, changing the incident field over the sensor.

and composite stiffness in the behaviour of the sensor, a 2D structural mechanics finite element model was built (COMSOL Multiphysics 5.2, COMSOL Inc., Sweden). A neo-Hookean hyperelastic material model [22] was used for the cilia. The model consists in the artificial cilia with a fixed constraint in their base as a boundary condition. In order to simulate the contact of each cilium with a plane, a hybrid geometry was used (see Fig. 4), in which the lower force range was modeled as a single pillar with a gaussian shaped force distributed over the pillar wall and concentrated near its top surface and the saturation force range with a rigid indentor placed initially over the cilium is lowered incrementally while deforming it. Once the simulation is complete, the deformation of 10 points evenly spaced over the cilia symmetry axis are exported, as well as the reaction force generated by the indentor over the pillar.

\section{B. Stray magnetic field}

The assumptions that the pillar is perfectly elastic and uniformly and isotropically magnetized in its symmetry axis, are made. In these conditions, the pillar can be approximated by a series of infinitesimal cross sections $S_{i}$, for which the magnetization of the non-deformed pillar is normal to the cross-section plane. When the pillar deforms, these cross sections are rotated and translated, but not deformed, and therefore, the magnetization vector is also rotated and its origin position is translated. In order to calculate these magnetization vectors, it is assumed that the pillar can be approximated by $N$ planes (Fig. 3). The perpendicular vector to a certain plane $i$ was assumed to be the average vector between the center of the planes $i-1$ and $i$, and planes $i$ and $i+1$, that is:

$$
\begin{array}{r}
\mathbf{v}_{\mathbf{i}-\mathbf{1}}=\overrightarrow{i-1, i} ; \quad \mathbf{v}_{\mathbf{i}+\mathbf{1}}=\overrightarrow{i, i+1} \\
\mathbf{v}_{\mathbf{i}}=\frac{\mathbf{v}_{\mathbf{i}+\mathbf{1}}+\mathbf{v}_{\mathbf{i}-\mathbf{1}}}{2}
\end{array}
$$

with $\mathbf{v}_{\mathbf{i}}$ representing the magnetization direction of all points over plane $i$. Finally the magnetization for a certain domain 


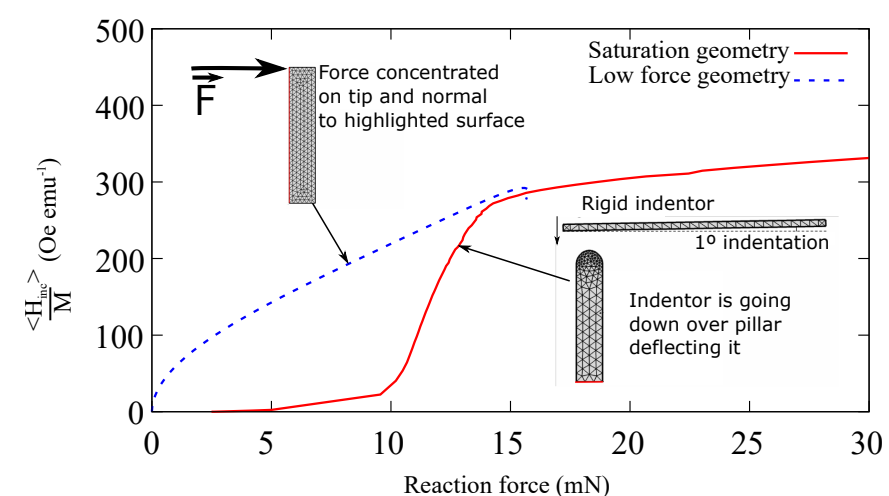

Fig. 4: Results of the simulation to determine the average field induced by an array of 5 pillars over a sensitive area of $3 \times 3 \mathrm{~mm}^{2}$. Insets: Geometry used for the simulation of each simulated geometry.

point $j$ in plane $i$ can be calculated from the total structure magnetization $\mathrm{M}$ and the total number of dipoles $N$ as $\mathbf{m}_{\mathbf{j}}=\frac{M}{N} \mid \mathbf{v}_{\mathbf{i}}$. Now that the magnetization over the structure domain is known, the magnetic field distributed over the GMR sensor must be calculated in order to determine its electrical resistance. Supposing that the active area of the sensor surface can be approximated as a grid with $k \times l$ elements, the magnetic field induced by a dipole $j$ over a surface element $(k, l)$ can be calculated as [24]:

$$
\mathbf{H}_{\mathbf{j}}\left(r_{j,(k, l)}\right)=\frac{1}{4 \pi}\left(\frac{3 \mathbf{r}_{\mathbf{j},(\mathbf{k}, \mathbf{l})}\left(\mathbf{m}_{\mathbf{j}} \cdot \mathbf{r}_{\mathbf{j},(\mathbf{k}, \mathbf{l})}\right)}{\left|\mathbf{r}_{\mathbf{j},(\mathbf{k}, \mathbf{l})}\right|^{5}}-\frac{\mathbf{m}_{\mathbf{j}}}{\left|\mathbf{r}_{\mathbf{j},(\mathbf{k}, \mathbf{l})}\right|^{3}}\right)
$$

with $\mathbf{r}_{\mathbf{j},(\mathbf{k}, \mathbf{l})}$ representing the vector pointing to the element $(k, l)$ and starting at dipole $j$ position. By adding all the contributions from all $N$ dipoles over a certain element $(k, l)$, and then averaging the magnetic field over all elements $(k, l)$, the average magnetic field over the spin-valve area is:

$$
\left\langle\mathbf{H}_{\text {inc }}\right\rangle=\frac{\sum_{k=1}^{N_{k}} \sum_{l=1}^{N_{l}} \sum_{j=1}^{N} \mathbf{H}_{\mathbf{j}}\left(r_{j,(k, l)}\right)}{N_{k} N_{l}}
$$

where $N_{k}$ and $N_{l}$ represents the number of elements in each surface direction.

With the incident field, and knowing the GMR sensor sensitivity, the output voltage $\mathrm{V}_{\text {out }}$ can be calculated by [25]

$$
\mathrm{V}_{\text {out }} \propto\left(\left\langle\mathbf{H}_{\text {inc }}\right\rangle \cdot \mathbf{e}_{\mathbf{v}}\right) \mathrm{I}_{\text {in }}
$$

where $I_{\text {in }}$ is the input current and $\mathbf{e}_{\mathbf{v}}$ is a unitary vector colinear with the spin-valve sensitive direction. This system was simulated with a C++ code with CUDA acceleration [23].

\section{Validation}

The model was verified against the results reported by [26], and validated against the experimental results reported in [27], obtaining in all cases a deviation lower than $15 \%$ between reported and simulated data. In Fig. 4, the results of the sensor simulation using both geometries is presented, for a sensor with 5 cilia, $3 \times 3 \mathrm{~mm}^{2}$ active area, each with $1 \mathrm{~mm}$ height and $200 \mu \mathrm{m}$ diameter and a Young's Modulus of $E=1 \mathrm{MPa}$. The point where both simulational results intersect each other will be roughly the saturation point of the sensor.

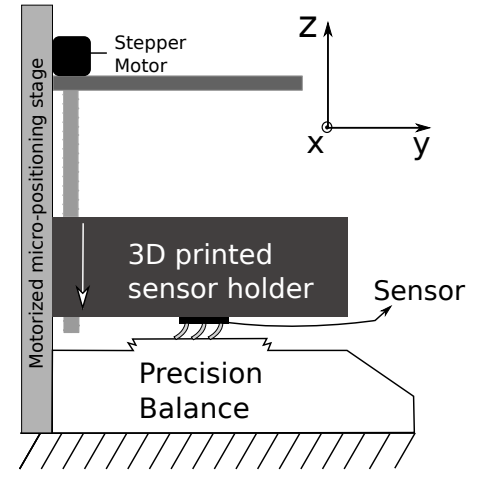

(a)

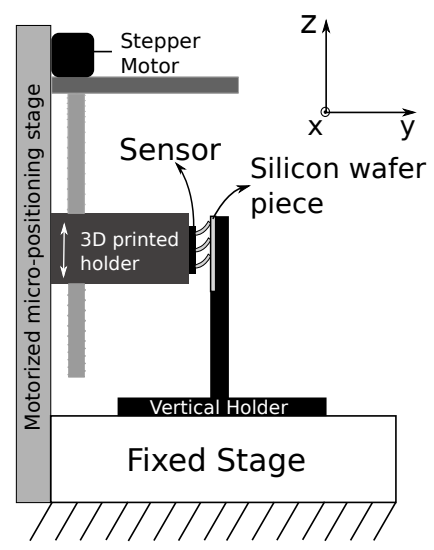

(b)

Fig. 5: Diagram of the setups used to perform sensor characterization. The dashed lines represent the setup base. a) Force characterization setup: A stepper motor is used to move a stage to which the sensor is fixed in such a way that the cilia will come in contact with the balance. b) Electrical stability and surface roughness setups: The vertical holder is positioned close enough to the micro-positioning setup to allow contact of the cilia with the holder.

\section{EXPERIMENTAL SETUP AND RESULTS}

\section{A. Force characterization}

To perform the force characterization of the sensor, a current source (Keithley 2401) and a multimeter (HP 34401A) controlled in real time via General Purpose Interface Bus (GPIB) were used, in addition to a motorized micro-positioning setup and a precision balance (Precisa junior 60A). The chipcarrier holding the sensor was connected to a self-adhesive mini-breadboard and glued to the bottom part of the micropositioner stage, in such a way that the cilia could be in contact with the balance plate (Fig. 5a). The stage was lowered until the cilia were fully deflected, and then moved away from the balance in incremental steps of $100 \mu \mathrm{m}$, until the cilia were no longer in contact.

The acquired data was compared to the simulation, by fitting the simulation results to the experimental ones, keeping as fixed parameters the pillar height of $1 \mathrm{~mm}$ and Young's Modulus of $1 \mathrm{MPa}$, and as free parameters, the pillar diameter and cilia magnetic moment. A linear fit was also performed in the low force regime obtained for each sensor to estimate 
TABLE I: Measured pillar diameter $\left(d_{m}\right)$ at the tip and the base, simulation fitting parameters (diameter $d_{s}$ and pillar magnetic moment $M_{\text {pillar }}$ ) and other experimental quantities for each value of pillar nominal diameter $d_{n}$

\begin{tabular}{|c|c|c|c|c|}
\hline $\begin{array}{c}d_{n} \\
(\mu)\end{array}$ & $\begin{array}{c}d_{m}(\text { base }) \\
(\mu \mathrm{m})\end{array}$ & $\begin{array}{c}d_{m} \text { (tip) } \\
(\mu \mathrm{m})\end{array}$ & $\begin{array}{c}\text { Min. force } \\
\text { feature }(\mu \mathrm{N})\end{array}$ & $\begin{array}{c}\text { GMR sensitivity } \\
\left(\mathrm{mOe} \mathrm{mV}^{-1}\right)\end{array}$ \\
\hline 120 & $\approx 250$ & $\approx 120$ & 333 & 100 \\
\hline 200 & $\approx 360$ & $\approx 200$ & 4067 & 100 \\
\hline \hline$d_{n}$ & $\begin{array}{c}d_{s} \\
(\mu \mathrm{m})\end{array}$ & $\begin{array}{c}M_{\text {pillar }} \\
(\mathrm{memu})\end{array}$ & $\begin{array}{c}\text { Sensitivity } \\
(\mathrm{mN} \mathrm{mV}-1\end{array}$ & $\begin{array}{c}\text { Saturation } \\
\text { force }(\mathrm{mN})\end{array}$ \\
\hline 120 & 200 & 0.3 & $9.62 \pm 1.25$ & 7.8 \\
\hline 200 & 280 & 0.7 & $16.7 \pm 4.2$ & 28.5 \\
\hline
\end{tabular}

the sensitivity, as well as a measurement of the diameter of the pillars at the tip and at the base using scanning electron microscopy photographs of the fabricated sensors.

The results obtained from the simulation, linear fitting, diameter measurements, saturation ranges and GMR sensor sensitivities are presented in table I, and a graphical representation of force vs voltage difference and the best fitting simulation results are presented in Fig. 6b. The simulated diameters agree with the ones measured, and the magnetic moment obtained from the fit are within the same order of magnitude reported in previously fabricated ciliary sensors $(\approx 1 \mathrm{memu})$ [18].

The minimum force feature detected with this setup (the force correspondent to the last stepper motor iteration before reaching the point of no contact with the balance, i.e. the minimum force measure in this experiment was limited by the stepper motor resolution) corresponds to $333 \mu \mathrm{N}$ for the pillars with diameter $\mathrm{d}=120 \mu \mathrm{m}$ while the sensor with diameter $\mathrm{d}=200 \mu \mathrm{m}$ registered a minimum force feature of $4067 \mu$ N. Fig. 6a shows the monitoring of the sensor response to realistic actuation, as random-oriented contact forces (that correspond to approximately $500 \mu \mathrm{N}$ of applied force) and then upon air flow (three direct blows over the sensor area). The power consumption of the sensor with a biasing current of $I_{\text {bias }}=450 \mu \mathrm{A}$ (used in all characterization tests) was 8.8 $\mathrm{mW}$.

\section{B. Electrical stability test}

An electrical stability test was performed for the sensor with cilia with nominal diameter $d_{n}=120 \mu \mathrm{m}$. The setup is shown in Fig. 5b. A fixed vertical holder with an atomically flat piece of silicon fixed to it is used and the breadboard with the sensor is glued to the stage in order to be parallel to the vertical holder. The micro-positioning setup was made to go up and down 20 $\mathrm{mm}$ cyclically, making the sensor range from a point of no contact, to a downward and upward dragging motion over the Si piece.

The results obtained for this test are presented in Fig. 6c. The amplitude of the performed cycles was fitted to a normal distribution, revealing a $2.39 \mathrm{mV}$ mean amplitude with a deviation of $0.21 \mathrm{mV}(8.8 \%)$ for a confidence interval of $95 \%$ during the test. A transient of $0.8 \mathrm{mV}$ is also observed, which is likely caused by parasitic capacitance within the
GMR sensor die, and imprecision in the movements of the micro-positioning setup.

\section{Noise study}

The noise detected by the sensor was acquired by placing the sensor within an armored container (to minimize external electromagnetic interference contributions to the measured values) and powered using $8 \mathrm{~V}$ provided by a battery pack. Then, a spectrum analyzer measured the sensor voltage and calculated its RMS voltage noise density in the frequency domain.

The resulting spectrum analyzer output is presented in figure $6 \mathrm{~d}$ ), in which the values of detectivity were calculated using the sensor with higher experimentally obtained sensitivity (the sensor with $d_{n}=120 \mu \mathrm{m}$, with a sensitivity of $3.85 \pm 0.49 \mathrm{mN}$ $\mathrm{mV}^{-1}$ for an $8 \mathrm{~V}$ powered sensor). From the noise spectral density, it can be observed that sensor noise at $5 \mathrm{~Hz}$ is 4.00 $\mu \mathrm{V} / \sqrt{\mathrm{Hz}}$, corresponding to a detectivity of $1.04 \mu \mathrm{N} / \sqrt{\mathrm{Hz}}$. For a sampling rate of $100 \mathrm{~Hz}(50 \mathrm{~Hz}$ bandwidth), the noise floor is at $9.8 \mu \mathrm{V}$, corresponding to $2.45 \mu \mathrm{N}$. The difference between this value and the experimental uncertainties obtained in subsection IV-A $(\approx 40 \mu \mathrm{V})$ are likely due to external electromagnetic intereference and the experimental setup used. It should also be noted that the sensor loses its effectiveness near strong magnetic fields (larger than $20 \mathrm{Oe}$, by reaching the GMR sensor saturation domain), however, no signs of severe impairement near unmagnetized ferromagnetic materials were observed.

\section{CONCLUSIONS}

This paper focused on the design, simulation and characterization of a miniaturized light force sensor. A biomimetic approach was taken on the ciliary structure as an array of soft, permanently magnetized pillars over a GMR sensor.

The fabrication process of the sensor is presented, as well as a simulation model, that fitted well with experimental data over the whole force sensing domain of this sensor.

Characterization tests performed to the sensor achieved a minimum detectable force feature of $333 \mu \mathrm{N}$, and the detectivity at $5 \mathrm{~Hz}$ was measured to be $1.04 \mu \mathrm{N} / \sqrt{\mathrm{Hz}}$. Further improvement of this sensor includes the integration of the magnetized cilia over a flexible substrate, to allow assembly over non-flat supports, as well as the development of a matrix, that would allow the detection of multiple points of contact simultaneously.

\section{REFERENCES}

[1] R.S. Dahiya. P. Mittendorfer, M. Valle, G. Cheng and V. J. Lumelsky, Directions towards effective utilization of tactile skin: A Review, in IEEE Sensors journal, pp. 4121-4138, Nov. 2013

[2] M. Cutkosky, R. Howe, W. Provancher, Force and Tactile Sensors, in Springer Handbook of Robotics, Springer, pp. 455-475

[3] M. Tiwana, S. Redmont and N. Lovell, A review of tactile sensing technologies with applications in biomedical engineering, in Sensors and Actuators A: Physical, vol 179, pp. 17-31, Jun. 2012

[4] M. Strohmayr, H. Saal, A. Potdar, P. van de Smagt, The DLR touch sensor I: A flexible tactile sensor for robotic hands based on a crossedwire approach, in Intl. Conf. Intelligent Robots and Systems, pp. 897903 , Oct. 2010 
a)

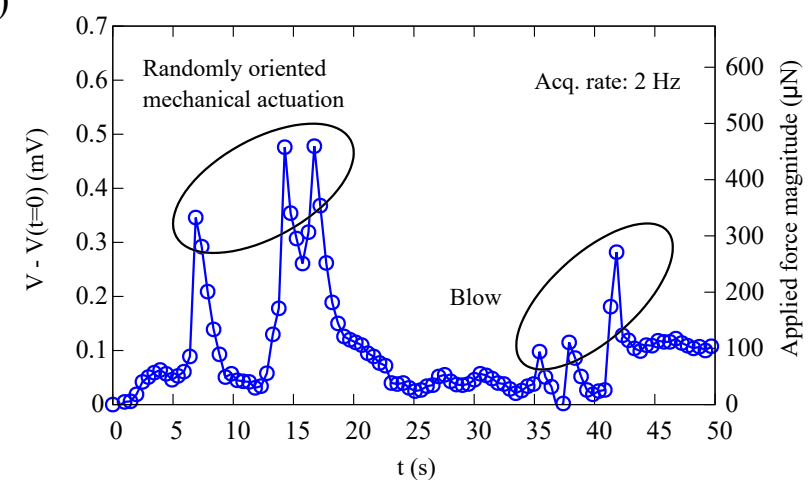

c)

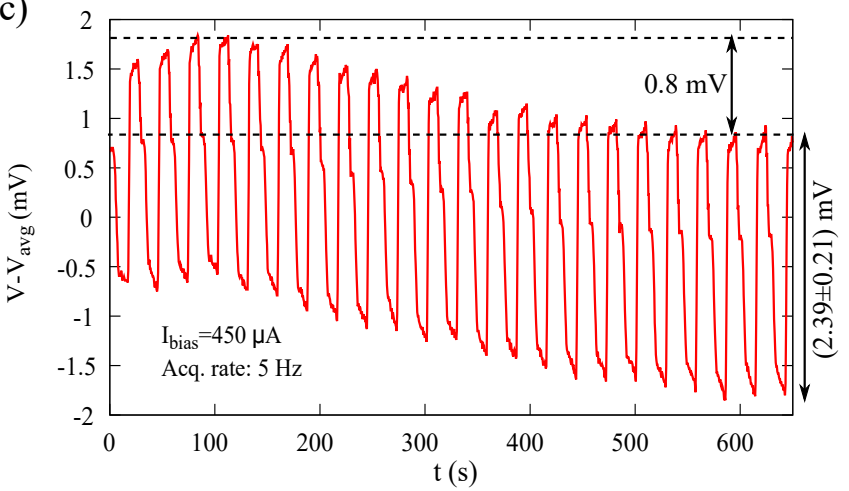

b)

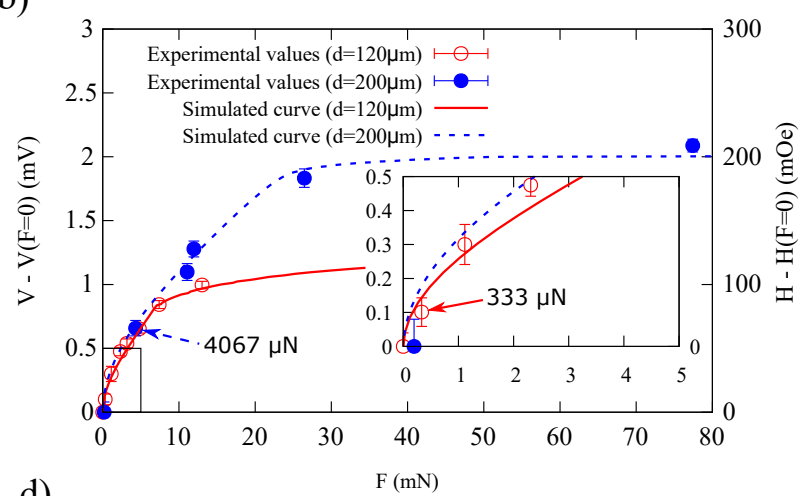

d)

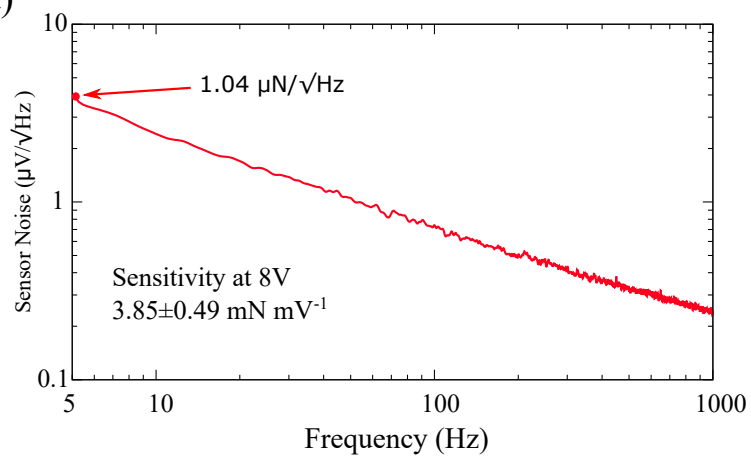

Fig. 6: Results obtained for the various characterization tests performed. $t$ represents acquisition time, $F$ represents applied force and $V$ represents measured voltage ( $\mathrm{V}_{\text {avg }}$ represents average voltage over total acquisition time). a) Results of a test performed using the sensor with $\mathrm{d}=120 \mu \mathrm{m}$ in which a randomly oriented force was applied to the sensor (until $t=25$ seconds), and then, from $t=35$ seconds, three separate blows. b) Force characterization test results for both tested pillar diameters and respective simulated curves, with parameters presented in table I. Inset: Plot zoom between $F=0 \mathrm{~N}$ and $F=5 \mathrm{mN}$. c) Results of the electrical stability test. d) Noise figure of the fabricated sensor, as a function of frequency. The presented 1.04 $\mu \mathrm{N} / \sqrt{\mathrm{Hz}}$ detectivity is relative to the fabricated sensor with higher sensitivity (with nominal diameter of $120 \mu \mathrm{m}$ ).

[5] N. Jamali, M. Maggiali, F. Giovanni, G. Metta, L. Natale, A new design of a findertip for the iCub hand, in Intl. Conf. on Intelligent Robots and Systems, pp. 2705-2710, Sep. 2015

[6] H. Lee, J. Chung, S. Chang, E. Yoon, Normal and Shear forces measurement using a flexible polymer tactile sensor with embedded multiple capacitors, in J. Microelectromechanical Systems, pp. 934-942, Aug. 2008

[7] M. Ohka, H. Kobayashi, J.Takata, Y. Mitsuya and H. Yussof, A robotic finger equipped with an opticl three-axis tactile sensor, in Itnl. Conf. Robotics and Automation, pp. 3425-3430, May 2008

[8] A. Cirillo, P. Cirillo, G. De Maria, C. Natale and S. Pirozzi, An Artificial Skin Based on Optoelectronic Technology, Sensors and Actuators A: Physical, vol. 212, pp. 110-122, Mar. 2013

[9] R. S. Dahiya, L. Lorenzelli, G. Metta, M. Valle POSFET devices based tactile sensing arrays, in Intl. Symp. Circuits and Systems, pp. 893-896, May 2010

[10] L. Jamone, L. Natale, G. Metta and G. Sandini, Highly sensitive soft tactile sensors for an anthropomorphic robotic hand. IEEE Sensors Journal, Vol. 15, No. 8, pp. 4226-4233. 2015

[11] T. P. Tomo, S. Somlor, S. Schmitz, L. Jamone, W. Huang, H. Kristanto and S. Sugano, Design and Characterization of a Three-Axis Hall EffectBased Soft Skin Sensor. MDPI Sensors, Vol. 16, No. 4, pp. 491. 2016

[12] M. Balicki, A. uneri, I. Iordachita, J. Handa, P. Gehlbach, R. Taylor, Micro-force sensing in robot assisted membrane peeling for vitreoretinal surgery, Med. Image Comput. Comput. Assist. Interv., vol. 13, pp. 303310,2010

[13] T. Keil, Functional morphology of insect mechanoreceptors, in Microscopy Research and Technique, 1997

[14] Y. Tang, R. L. Peterson and K. Najafi, Technology for fabricating dense 3-D microstructure arrays for biomimetic hair-like sensors, in Intl. Conf. on Micro Electro Mechanical Systems, pp. 355-358, Jan. 2013

[15] F. Li, W. Liu, C. Stefanini, X. Fu, and P. Dario, A Novel Bioinspired
PVDF Micro/Nano Hair Receptor for a Robot Sensing System, Sensors (Basel, Switzerland), vol. 10, no. 1, pp. 944-1011, Jan. 2010

[16] A. Virta, J. Timonen, R. Ras and Q. Zhou, Force sensing using artificial magnetic cilia, in Intl. Conf. Intelligent Robots and Systems, pp. 7-12, Oct. 2012

[17] A. Alfhadel, B. Li, A. Zaher, O. Yassine and J. Kosel, A magnetic nanocomposite for biommimetic flow sensing, in Lab on a Chip, vol. 14, pp. 4362-4369, Aug. 2014

[18] A. Alfhadel, M. Khan, S. Cardoso, J. Kosel, A single magnetic nanocomposite cilia force sensor, in IEEE Sensors - Applications Symposium, Apr. 2016

[19] V. Gehanno, P. Freitas, A. Veloso, J. Ferrira, B. Almeida, J. Sousa, A. Kling, J. Soares and M. da Silva, Ion beam deposition of Mn-Ir spin valves, in IEEE T. Magnetics, v. 35, pp 4361-4367, Sep. 1999

[20] D. Leitão, J. Amaral, S. Cardoso, C. Reig, Microfabrication Techniques, in Giant Magnetoresistance (GMR) Sensors, Springer, pp. 31-45, 2013

[21] E. Carrera, G. Giunta and M. Petrolo, Beam Structures: Classical and Advanced Theories, John Wiley \& Sons, 2011

[22] R. Ogden, Non-linear Elastic Deformations, Courier Corporation, 1997

[23] J. Sanders and E. Kandrot, CUDA by Example: An Introduction to General-Purpose GPU Programming, Addison-Wesley, 2010

[24] D. Dunlop, O. Ozdemir, Rock Magnetism, Cambridge University Press, 1997

[25] P. Freitas, R. Ferreira, S. Cardoso and F. Cardoso, Magnetoresistive Sensors, in J Phys.: Condens. Matter, v.19, pp. 165221-165242, Apr. 2007

[26] J. Mann, R. Lam, S. Weng, Y. Sun, J. Fu, A silicone-based stretchable micropost array membrane for monitoring live-cell subcellular cytoskeletal response, in Lab on A Chip, vol. 4, pp. 731-740, Dec. 2011

[27] R. Parween and R. Pratap, Modelling of soldier fly halteres for gyroscopic oscillations., Biology Open, 2015 